\title{
Evaluación de las competencias de trabajo en equipo y liderazgo y la comunicación efectiva en la asignatura de Distribución de la información espacial del MUIGG.
}

\author{
Eloina Coll Aliaga a , José Carlos Martínez LLario ${ }^{a}$ \\ a Departamento de Ingeniería Cartográfica, Geodesia y Fotogrametría (ecoll@cgf.upv.es), \\ (jomarlla@cgf.upv.es)
}

\begin{abstract}
This paper presents a real case used in the subject of master's degree for the evaluation of the transversal competences of leadership and team work and effective communication. The students develop these cross-disciplinary competences as well as the specific competences of the subject by acquiring a better understanding of the real work environment.
\end{abstract}

Keywords: leadership, teamwork, effective communication. Inspire

\begin{abstract}
Resumen
Este trabajo presenta un caso real utilizado en la asignatura de máster para la evaluación de las competencias transversales de liderazgo y trabajo en equipo y comunicación efectiva. Los estudiantes desarrollan dichas competencias transversales a la vez que las competencias específicas de la materia adquiriendo una mejor comprensión del entorno de trabajo real.
\end{abstract}

Palabras clave: liderazgo, trabajo en equipo, comunicación efectiva. Inspire

\section{Introducción}

La asignatura de Distribución de la Información Espacial es una asignatura troncal del Máster Universitario en Ingeniería en Geomática y Geoinformación de la Universitat Politècnica de València que cuenta con 6 créditos ECTS, (3 teóricos y 3 prácticos). Esta asignatura se centra en que los estudiantes sean expertos en las especificaciones de datos de la Directiva Europea Inspire. Se trabaja con el Marco Normativo de la información espacial. Se estudia el modelo genérico conceptual de Inspire y las especificaciones de datos. Se realiza la gestión de los modelos de datos utilizando bases de datos espaciales, operaciones de análisis espacial según especificación OGC (Open Geospatial Consortium) y se implementan modelos de datos cartográficos Inspire con postgreSQL y postGIS. Estos conocimientos técnicos son altamente demandados en los puestos de trabajos en los que se requieren técnicos especialistas en Geoinformación. 
En la actualidad, las empresas buscan estudiantes que además de ser buenos técnicamente, presenten otras habilidades, es decir, los estudiantes necesitan además de las competencias específicas, adquirir competencias transversales (CT) y para ello se debe utilizar un enfoque del aprendizaje basado en competencias y hacer entender al estudiante que es fundamental que se incorporen técnicas y métodos nuevos para que puedan aplicar los conocimientos a situaciones nuevas y puedan integrar las actitudes y valores para afrontar nuevos retos (Villa, 2011).

El aprendizaje basado en competencias ha supuesto una sustancial transformación de la docencia universitaria, tanto en lo referente a las metodologías empleadas como en la implantación de sistemas de evaluación y control de su calidad (Hermosilla, 2013). Para conseguir este aprendizaje la Universitat Politècnica de València (UPV) desarrolla, desde el curso 2013-14, un proyecto institucional que define 13 CT (ICE-UPV 2018) y tiene como objetivo principal que los egresados de cualquier título oficial impartido en esta universidad acrediten dichas CT. En dicho Proyecto se establecen actividades formativas y pautas para su evaluación a base de rúbricas y es la Entidad Responsable del Título (ERT) la que se encarga de repartir dichas competencias entre las asignaturas para que se trabajen a la vez que se imparte la docencia.

\subsection{Objetivos}

En el curso 2014-2015 se imparte por primera vez esta asignatura y se combina la docencia de clase magistral con algunas clases en las que se desarrollan trabajos colaborativos entre los alumnos y el profesor para estudiar una posible implementación de una especificación técnica. Se plantea que algunos alumnos expliquen los resultados obtenidos al resto de la clase y se observa que es una experiencia por la que deberían pasar todos los alumnos y que conociendo unas normas preestablecidas para realizar una presentación oral, sería fácil realizar una evaluación entre ellos. Al siguiente curso (2015-2016) desde la ERT se plantea que las asignaturas sean puntos de control de dos de las trece competencias transversales y se asignan a esta asignatura las competencias de liderazgo y trabajo en grupo y comunicación efectiva.

Es durante este curso acádemico, cuando se decide que todos los alumnos deben preparar en grupo una especificación técnica durante las clases presenciales y realizar una exposición publica donde todos ellos puedan ser evaluados por sus compañeros de la competencia oral de la comunicación efectiva, para ello se utilizan rúbricas de coevaluación y autoevaluación (De Miguel, 2006) conocidas desde el primer día de clase. Además se utilizan las rúbricas que han sido desarrolladas por los grupos de trabajo del ICE (https://www.upv.es/entidades/ICE/info/U0724624.pdf).

El objetivo perseguido es que el alumno adquiera las competencias transversales que son punto de control a la vez que las especificas. Para la comunicación efectiva, hay que tener en cuenta la parte oral y la parte escrita y para el trabajo en grupo y liderazgo hay que organizar actividades en las que los alumnos trabajen en grupo y que además demuestren el papel de lideres. Las competencias deben ser evaluadas durante el trascurso de la asignatura.

La presente comunicación pretende resumir esta experiencia docente, detallando las conclusiones a las que tanto profesores como estudiantes hemos llegado.

(cc) EY-NC-ND 2018, Universitat Politècnica de València

Congreso IN-RED (2018) 


\subsection{Procedimiento utilizado}

Durante el transcurso de la asignatura se van a realizar 6 trabajos en grupo (2 en la parte de teoría y 4 en la parte práctica), estos van a servir para valorar la competencia de liderazgo y trabajo en grupo. Se pretende que los estudiantes trabajen en diferentes grupos con criterios diferentes durante la asignatura y que sean capaces de obtener evidencias de dicha competencia. Para ello, deberán entregar actas de las sesiones de trabajo donde se explique el orden del día, los componentes de dicha reunión de trabajo y las conclusiones obtenidas durante la sesión.

Se realizan tres tareas durante las clases prácticas que se encuentran en el PoliformaT y que se realizan en grupo de 2 personas. Una de las tareas se realiza dentro de un módulo de aprendizaje (Fig, 1) donde existen objetos de aprendizaje que ayudan a los estudiantes a realizar más fácilmente las prácticas. Las prácticas son evaluadas por observación y por corrección de la memoria entregada en la tarea. Esta memoria servirá para evaluar la comunicación escrita.

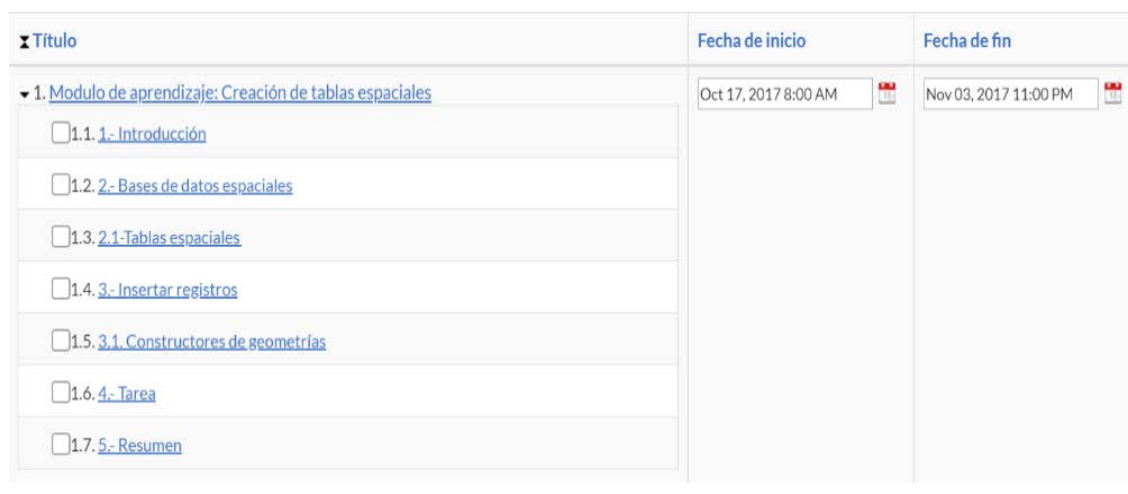

Fig. 1 Modulo de aprendizaje

Existen dos exposiciones públicas para adquirir la competencia de comunicación oral y al realizarse en grupo también sirve para evaluar el trabajo en grupo.

La primera se realiza en grupos de 4 personas elegidos por el profesor. En el PoliformaT existen presentaciones técnicas de formatos exigidos por el OGC para trabajar con información espacial y cada grupo elige una presentación. Durante una sesión de dos horas trabajan en el aula y al finalizar deben entregar un acta de la reunión realizada con las conclusiones sacadas del tema. Existe un día marcado en la temporización (fig. 2) donde expondrán el tema y serán evaluados con la rúbrica que se presenta en la tabla1 por parte de sus compañeros.

Dentro de la Directiva Inspire existen muchas especificaciones de datos. En una sesión de teoría se explica el modelo genérico de Inspire y se organizan los estudiantes en grupos de 5 personas para explicar una de las siguientes especificaciones de datos: Hidrografía, Redes 
Evaluación de las competencias de trabajo en equipo y liderazgo y la comunicación efectiva en la asignatura de Distribución de la información espacial del MUIGG.

de transporte, Unidades administrativas, Ocupación del suelo, Direcciones y nombres geográficos.

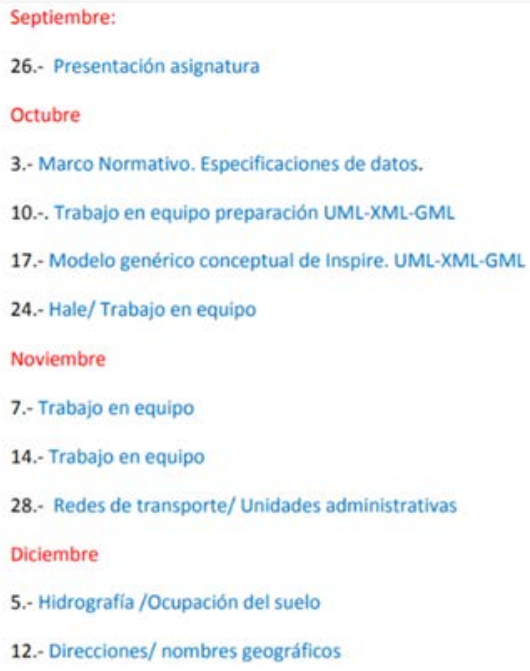

Fig. 2 Temporización de la asignatura

Los integrantes de estos equipos se eligen entre los estudiantes, así como la especificación que van a desarrollar. Durante tres sesiones de 2 horas se trabaja en clase para preparar la especificación y fuera del horario de la asignatura deben de realizar al menos dos reuniones más. En total deben entregar actas como mínimo de 5 reuniones, donde especificarán quien ha asumido el papel de líder y qué tareas han sido asignadas a cada uno de los miembros del grupo durante la sesión.

Las defensas de estas especificaciones están en la temporización de la asignatura y una de las condiciones que se pide es que se utilicen técnicas diferentes en cada presentación. Es decir, la dificultad de la especificación va de menor a mayor, ya que los conceptos generales se van a ir repitiendo, pero se exige que los recursos utilizados para el tema específico sea diferente (hacer un ejercicio práctico, un test, un kahoot, trabajar en equipo con diferentes cartografías, etc.). La complejidad técnica será menor, pero la confección de la presentación mayor.

Tabla 1. Rubrica utilizada para la coevaluación

\section{4}

3

\section{2}




\begin{tabular}{|c|c|c|c|c|}
\hline Pronunciación & $\begin{array}{l}\text { Habla despacio y } \\
\text { claro y vocaliza } \\
\text { bien }\end{array}$ & 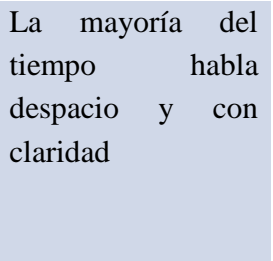 & $\begin{array}{l}\text { Unas veces } \\
\text { habla despacio } \\
\text { y con claridad } \\
\text { y otras es } \\
\text { difícil de } \\
\text { entender }\end{array}$ & $\begin{array}{l}\text { Habla rápido } \\
\text { o presenta } \\
\text { espacios sin } \\
\text { hablar. Con } \\
\text { muletillas }\end{array}$ \\
\hline Volumen & $\begin{array}{l}\text { Adecuado y lo } \\
\text { suficientemente } \\
\text { alto cuando } \\
\text { enfatiza } \\
\text { importante }\end{array}$ & $\begin{array}{l}\text { Levanta demasiado } \\
\text { la voz todo el } \\
\text { tiempo. }\end{array}$ & $\begin{array}{l}\text { Habla } \\
\text { demasiado bajo } \\
\text { al exponer y de } \\
\text { forma } \\
\text { monótona. }\end{array}$ & $\begin{array}{l}\text { El volumen } \\
\text { es muy débil, } \\
\text { casi no se le } \\
\text { oye }\end{array}$ \\
\hline $\begin{array}{l}\text { Postura del } \\
\text { cuerpo/contacto visual }\end{array}$ & $\begin{array}{lr}\text { La postura y los } \\
\text { gestos son muy } \\
\text { adecuados. } \\
\text { a todos los } \\
\text { compañeros con } \\
\text { naturalidad }\end{array}$ & $\begin{array}{lr}\text { La postura y los } \\
\text { gestos } & \text { son } \\
\text { adecuados pero } \\
\text { está apoyado en } \\
\text { algún lugar. Suele } \\
\text { mirar sólo a alguna } \\
\text { parte de la } \\
\text { audiencia. }\end{array}$ & $\begin{array}{l}\text { En ocasiones le } \\
\text { da la espalda a } \\
\text { sus } \\
\text { compañeros. } \\
\text { Algunas veces } \\
\text { mantiene la } \\
\text { postura } \\
\text { adecuada. }\end{array}$ & $\begin{array}{l}\text { No mantiene } \\
\text { la postura } \\
\text { apropiada de } \\
\text { una } \\
\text { exposición } \\
\text { oral y la } \\
\text { mayoría de } \\
\text { las veces no } \\
\text { mira a sus } \\
\text { compañeros. }\end{array}$ \\
\hline Contenido & $\begin{array}{l}\text { Demuestra un } \\
\text { completo } \\
\text { entendimiento } \\
\text { del tema. }\end{array}$ & $\begin{array}{l}\text { Demuestra un buen } \\
\text { entendimiento del } \\
\text { tema. }\end{array}$ & $\begin{array}{l}\text { Demuestra } \\
\text { entendimiento } \\
\text { de partes del } \\
\text { tema. }\end{array}$ & $\begin{array}{l}\text { No parece } \\
\text { entender } \\
\text { muy bien el } \\
\text { tema. }\end{array}$ \\
\hline Vocabulario & $\begin{array}{l}\text { Usa vocabulario } \\
\text { técnico que } \\
\text { enriquece a la } \\
\text { audiencia. }\end{array}$ & $\begin{array}{ll}\text { Usa vocabulario } \\
\text { técnico e incluye } \\
\text { algunas palabras } \\
\text { nuevas para la } \\
\text { mayor parte de la } \\
\text { audiencia }\end{array}$ & $\begin{array}{l}\text { Usa } \\
\text { vocabulario } \\
\text { apropiado y } \\
\text { conocido por la } \\
\text { audiencia. }\end{array}$ & $\begin{array}{l}\text { No usa } \\
\text { vocabulario } \\
\text { técnico y } \\
\text { repite } \\
\text { muchas } \\
\text { veces las } \\
\text { mismas } \\
\text { palabras }\end{array}$ \\
\hline $\begin{array}{l}\text { Comprensión/secuencia } \\
\text { ción }\end{array}$ & $\begin{array}{lr}\begin{array}{l}\text { Presenta } \\
\text { conexión }\end{array} \text { una } \\
\text { slguida, el } \\
\text { lógico en } \\
\text { exposición } \\
\text { contesta } \\
\text { preguntas } \\
\text { planteadas. }\end{array}$ & $\begin{array}{l}\text { Puede contestar } \\
\text { algunas preguntas y } \\
\text { la conexión entre } \\
\text { las diapositivas es } \\
\text { bastante buena, }\end{array}$ & $\begin{array}{l}\text { Realiza muy } \\
\text { poco la } \\
\text { conexión entre } \\
\text { las diapositivas } \\
\text { y contesta muy } \\
\text { poco a las } \\
\text { preguntas }\end{array}$ & 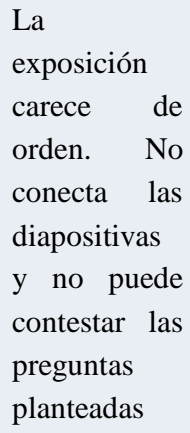 \\
\hline
\end{tabular}


Evaluación de las competencias de trabajo en equipo y liderazgo y la comunicación efectiva en la asignatura de Distribución de la información espacial del MUIGG.

Los grupos son evaluados utilizando la tabla 2. Cada alumno rellena su nombre y evalúa a los 4 compañeros que aparecen en la tabla.

Tabla 2. Tabla que rellenan los estudiantes en la coevaluación.

\begin{tabular}{|l|l|l|l|l|}
\hline Alumno: & Nombre1 & Nombre2 & Nombre3 & Nombre4 \\
\hline Pronunciación & & & \\
\hline Volumen & & & \\
\hline Postura del cuerpo/contacto & & \\
visual
\end{tabular}

El tercer trabajo en grupo es totalmente práctico y se realiza en grupo de 2 personas. Se trata de realizar la implementación de una especificación de datos utilizando bases de datos espaciales. Se realiza durante 5 sesiones de 2 horas, se entrega el código implementado y la defensa es individual.

\subsection{Resultados}

En la figura 3 se muestran los ítems de evaluación donde se evalúan a la vez las competencias específicas y las transversales: 3 trabajos prácticos en grupo 30\%. Presentación por grupos de la especificación técnica y entrega de las actas de las reuniones realizadas 30\%. Coevaluación alumno profesor 10\% e Implementación del modelo de datos $30 \%$. Entrega de la memoria y defensa sobre el ordenador del laboratorio donde se tenga instalado el modelo.

\begin{tabular}{|c|c|c|c|c|c|c|c|c|}
\hline Titulo* & & Media" & Fecha de entrega & Publicada para estudiantes & Incluido en la nota final & Ordenar & ndo $=$ & Editor de notas'". \\
\hline Ejercicios SOL & Editar & $9 / 10$ & 03-nov-2017 & s & Si & 1 & $\bar{\nabla}$ & desde Tareas \\
\hline creación de tablas & $\underline{\text { Editar }}$ & $9 / 10$ & 10-nov-2017 & s & Si & $2=$ & $=\mp$ & desde Tareas \\
\hline Creation of spatial tables & $\underline{\text { Editar }}$ & $9 / 10$ & 10-nov-2017 & $\mathrm{s}$ & Si & 3 & $=$ & desde Tareas \\
\hline Relaciones yoperadores espaciales & $\underline{\text { Editar }}$ & $9 / 10$ & 11-ene-2018 & s & Si & 4 & $=\mp$ & desde Tareas \\
\hline relations and spatial operators & $\underline{\text { Editar }}$ & 9/10 & 11 -ene-2018 & s & Si & 5 & $=\mp$ & desde Tareas \\
\hline coevaluación & $\underline{\text { Editar }}$ & $7 / 10$ & & s & Si & 6 & $=$ & \\
\hline Implementación modelo & $\underline{\text { Editar }}$ & $21 / 30$ & - & s & Sí & $7=$ & $=$ & \\
\hline presentacion & Editar & $22 / 30$ & - & $\mathrm{s}$ & $\mathrm{Si}$ & 8 & 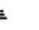 & \\
\hline
\end{tabular}

Fig. 3 Resumen de ítems de calificación (PoliformaT)

(cc) EY-NC-ND 2018, Universitat Politècnica de València

Congreso IN-RED (2018) 
Existe una coevaluación por parte de los estudiantes y del profesor (Fig. 4)

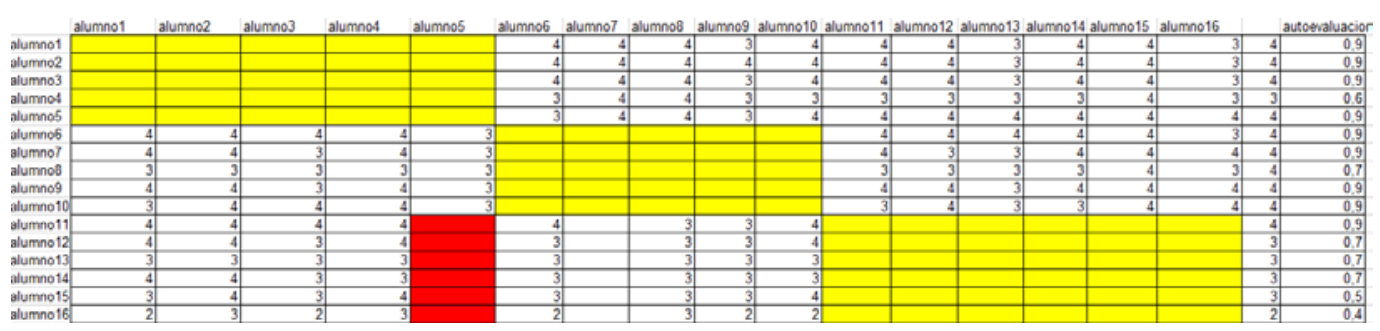

Fig. 4 Coevaluación de alumnos curso 2017-18

Al finalizar la asignatura se realiza una autoevaluación genérica (Fig. 5) donde el alumno evalúa su aportación en clase, su implicación en el trabajo en grupo y en las actividades individuales. Comenta su satisfacción personal y la valoración global de la asignatura.

Al finalizar el curso: Autoevaluación genérica

\begin{tabular}{|l|l|}
\hline & Puntos (1-10) \\
\hline 1. Participación y aportaciones en clase & \\
\hline $\begin{array}{l}\text { 2. Implicación en el trabajo en grupo (organización, } \\
\text { planificación, cumplimiento, participación, } \\
\text { aportaciones...) }\end{array}$ & \\
\hline 3. Capacidad de trabajo cooperativo & \\
\hline $\begin{array}{l}\text { 4. Implicación en las actividades individuales(organización, planificación, } \\
\text { cumplimiento...) }\end{array}$ & \\
\hline 5. Dedicación de tiempo a las diferentes actividades & \\
\hline 6. Apropiación de los contenidos trabajados & \\
\hline 7. Relaciones entre los miembros del grupo de trabajo & \\
\hline 8. Seguimiento de las tutorías & \\
\hline 9. Satisfacción personal & \\
\hline 10. Valoración GLOBAL & \\
\hline
\end{tabular}

Fig. 5 Autoevaluación genérica

\section{Conclusiones}

La principal conclusión que se obtiene, es que los estudiantes desde el inicio de la asignatura aceptan la evaluación conjunta de las competencias específicas y transversales.

$\mathrm{Al}$ tener que realizar varias exposiciones y tener que evaluar al resto de compañeros, hace que las clases sean más productivas.

En la asignatura se realiza un viaje de prácticas al Instituto Geográfico Nacional, que es el encargado de llevar a cabo la transformación de los datos espaciales a las especificaciones técnicas de Inspire y resulta altamente satisfactorio comprobar que los estudiantes se encuentran a la altura del lenguaje técnico utilizado por los responsables de estos trabajos. Recibimos felicitaciones del nivel que tienen y esto es debido a que trabajan durante 5 
sesiones profundamente con una especificación y luego la exponen al resto de sus compañeros. Además evalúan las exposiciones de los otros grupos de trabajo y finalmente realizan la implementación del modelo de datos de una especificación no vista en clase en una base de datos espacial.

Para facilitar el trabajo de los estudiantes se han desarrollado objetos de aprendizaje y módulo de aprendizaje que han sido utilizados para desarrollar la parte práctica.

El rendimiento académico de la asignatura es muy bueno, el 90\% de los estudiantes adquieren un nivel alto de las competencias que son punto de control y están muy contentos con el tipo de docencia recibida.

\section{Referencias.}

ICE-UPV (2018). Portal de competencias transversales de la Universitat Politècnica de València. http://www.upv.es/contenidos/COMPTRAN/ [Online; acceso 23-Marzo-2018].

https://www.upv.es/entidades/ICE/info/U0724624.pdf

HERMOSILLA Z., CLEMENTE M., TRINIDAD A., ANDRÉS J. (2013) “Competencia en comunicación oral: un reto para el ingeniero" En Garrigos et al. "New changes in technology and innovation.” INNODOCT 2013. Universidad Politècnica de Valencia.

DE MIGUEL, M. (2006) “Metodologías de enseñanza para el desarrollo de competencias. Orientaciones para el profesorado universitario ante el Espacio Europeo de Educación Superior.” en Educatio siglo XXI, Vol 24, pp 207-210.

VILLA SÁNCHEZ A., POBLETE RUIZ M. (2011) "Evaluación de competencias genéricas: principios, oportunidades y limitaciones” Bordón, Vol 63 Nº, pp147-170. 